\title{
Magnetic resonance assessment of aortic and mitral regurgitation
}

\author{
S R UNDERWOOD, R H KLIPSTEIN, D N FIRMIN, K M FOX, \\ P A POOLE-WILSON, R S O REES, D B LONGMORE
}

From the National Heart and Chest Hospitals, London

SUMMARY Magnetic resonance imaging provides an accurate method for the measurement of left and right ventricular volume. The ratio of left ventricular stroke volume to right ventricular stroke volume was calculated from contiguous transverse magnetic resonance images and was used to measure the severity of regurgitation in 18 patients with aortic regurgitation and 10 with mitral regurgitation. Cardiac anatomy was well demonstrated, allowing an assessment of relative chamber volumes and associated abnormalities, although valve abnormality was not well seen. There was a weak correlation between magnetic resonance measurements of left ventricular end diastolic volume and stroke volume ratio. The stroke volume ratio differed significantly in four groups with increasing angiographic severity of regurgitation, and all but the group with trivial regurgitation differed significantly from normal. There was good correlation between magnetic resonance and radionuclide measurements of left ventricular ejection fraction and stroke volume ratio, although the stroke volume ratio was consistently overestimated by radionuclide ventriculography. Correlation was less good for the right ventricular ejection fraction, which was underestimated by radionuclide ventriculography.

It is concluded that magnetic resonance imaging provides valuable information in patients with valvar regurgitation, and serves as a suitable standard by which to judge conventional techniques.

Magnetic resonance imaging now has an established place in diagnostic imaging of the brain and spinal cord. Its value lies in high soft tissue contrast and high resolution and in the fact that contrast is determined partly by the biochemical environment of the protons forming the image. Moving organs in the thorax and abdomen require special techniques to minimise artefact, but images of the heart with excellent anatomical definition can be produced with electrocardiographic gating alone. ${ }^{-3}$ Contrast media are not required because of the natural contrast between myocardium and flowing blood which usually gives no magnetic resonance signal.

Functional and anatomical information are obtained, and we have previously demonstrated that cardiac chamber volumes can be measured with an accuracy of approximately $2 \% \cdot{ }^{4}$ Ventricular volume measurements allow an assessment of systolic ven-

Requests for reprints to Dr S R Underwood, Magnetic Resonance Unit, The National Heart and Chest Hospitals, 30 Britten Street, London SW3 $6 \mathrm{NN}$.

Accepted for publication $10 \mathrm{June} 1986$ tricular performance (end systolic volume and ejection fraction), and the ratio of left ventricular stroke volume to right ventricular stroke volume gives a measure of valvar regurgitation and atrial shunting. ${ }^{5}$ In this study we have used measurements of left and right ventricular stroke volume to quantify the severity of aortic and mitral regurgitation and we have compared these findings with measurements made by radionuclide ventriculography and cardiac catheterisation.

\section{Patients and methods}

PATIENTS

We studied 28 patients ( 17 male, 11 female, mean age 53.5 (range 18-75)). Eighteen had isolated aortic regurgitation and 10 had isolated mitral regurgitation. The aetiology of aortic valve disease was unknown in nine, a bicuspid valve in two, Marfan's syndrome in one, ankylosing spondylitis in one, rheumatic valve disease in one, leaking homografts in three, and a leaking pulmonary valve autograft in one. The aetiology of mitral regurgitation was valve 
prolapse in seven, Marfan's syndrome in one, hypertrophic cardiomyopathy in one, and coronary artery disease in one. All patients were in sinus rhythm except four with mitral regurgitation who were in atrial fibrillation.

All patients were studied by magnetic resonance, 26 underwent cardiac catheterisation, and 26 equilibrium radionuclide ventriculography, investigations in each patient being completed within 8 weeks.

\section{MAGNETIC RESONANCE}

A Picker International Vista MR2055 magnetic resonance scanner operating at $0.5 \mathrm{~T}$ was used to acquire multiple $10 \mathrm{~mm}$ contiguous sections in the transverse plane at end diastole and at end systole. The images were reconstructed by two dimensional Fourier transformation from two averages of 128 spin echo sequences (echo time $24 \mathrm{~ms}$ ). Diastolic images were acquired immediately after the $R$ wave of the electrocardiogram, and systolic images were timed to coincide with the end of the $T$ wave. Diastole at one level was acquired at the same time as systole at another, and each pair of images took between 3 and 4 minutes to acquire, depending upon heart rate. Preliminary coronal images were acquired in the plane of the aortic valve and sagittal images in the plane of the pulmonary valve; 12 transverse images were usually sufficient to cover both ventricles. Total imaging time was between 40 and 60 minutes.

Left and right ventricular endocardial contours were assigned manually by use of a Nodecrest V77 computer, and diastolic and systolic volumes were measured by summing the areas of the chambers in each transverse image. The left and right ventricular stroke volumes, ejection fractions, and the left to right ventricular stroke volume ratio were derived from the volumes.

\section{RADIONUCLIDE VENTRICULOGRAPHY}

Equilibrium radionuclide ventriculography was performed after in vivo erythrocyte labelling with $740 \mathrm{MBq}$ of technetium-99m sodium pertechnetate. ${ }^{6}$ Sixteen frames were acquired in the left anterior oblique projection with $30^{\circ}$ of caudal tilt by means of a Siemens gamma camera $(200 \mathrm{~mm}$ field of view) and a Medical Data Systems " $A$ " computer. Fourier phase and amplitude images were constructed $^{7}$ and used together with the end diastolic and end systolic images to assign left and right ventricular regions of interest. Counts within these regions were used to calculate left and right ventricular ejection fractions and the left to right ventricular stroke volume ratio. Background counts were estimated from a crescentic region inferior and lateral to the left ventricle.

\section{CARDIAC CATHETERISATION}

Mitral and aortic regurgitation were assessed from an $x$ ray contrast left ventriculogram and aortogram respectively by one of three consultant radiologists without knowledge of the magnetic resonance or radionuclide findings. Aortic regurgitation was classified as trivial (I), mild (II), moderate (III), or severe (IV) according to whether regurgitant contrast opacified the left ventricle incompletely and was cleared at end systole (I), failed to be cleared at end systole (II), accumulated within the left ventricle with complete opacification (III), or filled the ventricle completely at the end of the first diastole following injection (IV). ${ }^{89}$ Mitral regurgitation was classified into the same grades according to the size of the regurgitant jet and the degree of left atrial opacification, taking into account the size of the left atrium. ${ }^{1011}$

\section{STATISTICAL ANALYSIS}

The Wilcoxon rank-sum test was used to compare measurements between groups and to compare with a group of normal subjects previously studied by the same methods. ${ }^{4}$

\section{Results}

Magnetic resonance image quality depended mainly upon the ability of the patient to keep still during acquisition and upon the cardiac rhythm. In the four patients with atrial fibrillation the images were of poorer quality but it was still possible to measure ventricular volume. Figure 1 shows a coronal image in one of the patients with atrial fibrillation and mitral regurgitation due to valve prolapse.

Cardiac anatomy was easily judged from the images, giving an estimate of relative chamber sizes, myocardial hypertrophy, and aortic dilatation. Figure 2 shows the dilated ascending aorta in a patient with Marfan's syndrome and mitral valve prolapse. In one patient with aortic regurgitation studied during an episode of infective endocarditis, an abscess mass was seen involving the anterior aortic wall compressing the right ventricular outflow tract (fig 3). This abnormality was also shown by echocardiography. The aortic and mitral valves were visible in many of the patients, particularly the mitral valve in the systolic images, but resolution was not sufficient to identify valve abnormalities.

Mean (SD) left ventricular end diastolic volume in the whole group of patients was $262 \mathrm{ml}(131.5)$, which is significantly higher $(\mathrm{p}<0.001)$ than the 


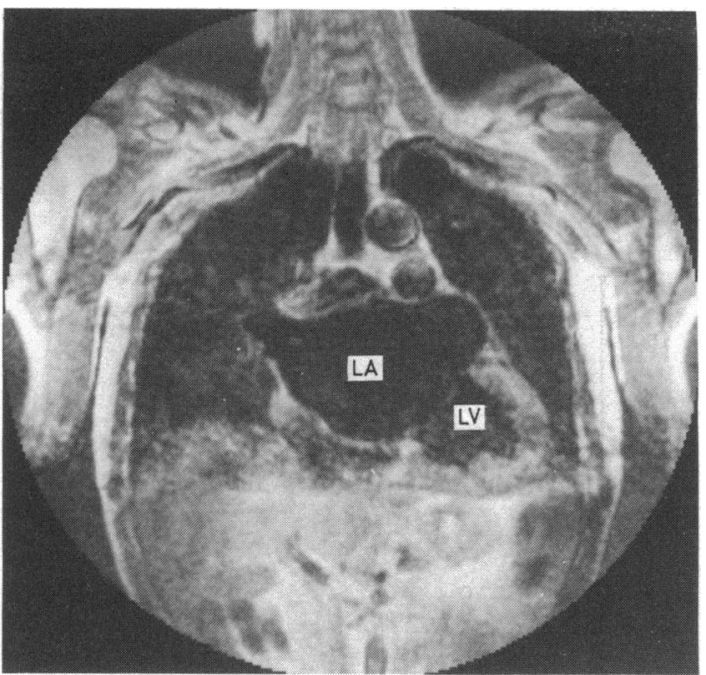

Fig 1 Systolic coronal magnetic resonance image through the left atrium ( $L A)$, mitral valve, and left ventricle $(L V)$ in a patient with atrial fibrillation and severe mitral regurgitation through a floppy mitral valve with ruptured chordae tendineae. The left atrium is very large and although the left ventricle is also enlarged (end diastolic volume $403 \mathrm{ml}$ ) systolic function was good (left ventricular ejection fraction 0.57). The regurgitant fraction was 0.47 .

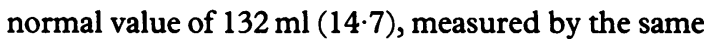
method in a group of 20 normal subjects. ${ }^{4}$ There was no significant difference $(p>0.05)$ between mean right ventricular end diastolic volume in the patients $(160 \mathrm{ml}(53.7))$ and the same group of normal subjects $(152 \mathrm{ml}(20.9))$. Figure 4 shows the correlation between left ventricular end diastolic volume and the left to right ventricular stroke volume ratio measured by magnetic resonance. There is a loose but significant correlation ( $r=0.75$, standard error of the estimate (SEE) $88.8 \mathrm{ml}, \mathrm{p}<0.001$ ).

Figure 5 compares magnetic resonance measurements of the stroke volume ratio with the semiquantitative assessment of severity of valvar regurgitation made at cardiac catheterisation. There was very little overlap between the four groups, and the mean stroke volume ratio increased with the severity of regurgitation. In the group with severe regurgitation, there was a wide range of stroke volume ratios (1.6-3.5), but in the other groups the ranges were narrower.

The mean (SD) values of stroke volume ratio in each group were: trivial $0.95(0.11)$, mild 1.27. $(0.09)$, moderate $1.50(0 \cdot 10)$, severe $2.34(0.50)$. Mean (SD) stroke volume ratio in 20 normal subjects who had been studied previously was $1.01(0.067),{ }^{4}$ so that although there was no significant difference between normal and the group with trivial regurgitation ( $p>$ 0.05 ), in the other three groups the difference in

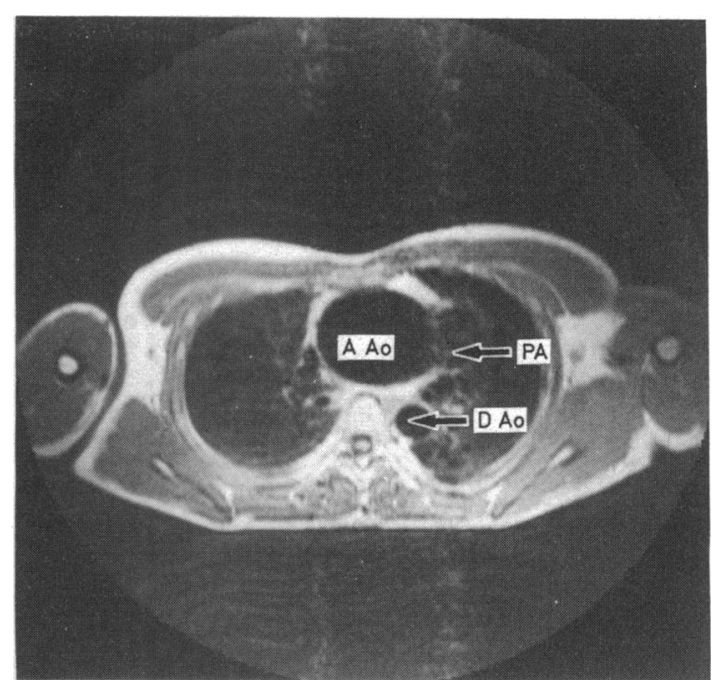

Fig 2 Magnetic resonance image of transverse section at the level of the pulmonary valve in a patient with Marfan's syndrome and mitral regurgitation through a floppy mitral valve. The ascending aorta $(A A o)$ is dilated $(67 \mathrm{~mm}$ diameter) and there is mild pectus excavatum. The aortic valve was competent. $D A o$, descending aorta; $P A$, pulmonary artery.

mean stroke volume ratio was highly significant $(\mathrm{p}<$ 0.001 ). The differences between the groups with trivial and mild regurgitation and between those with mild and moderate regurgitation were

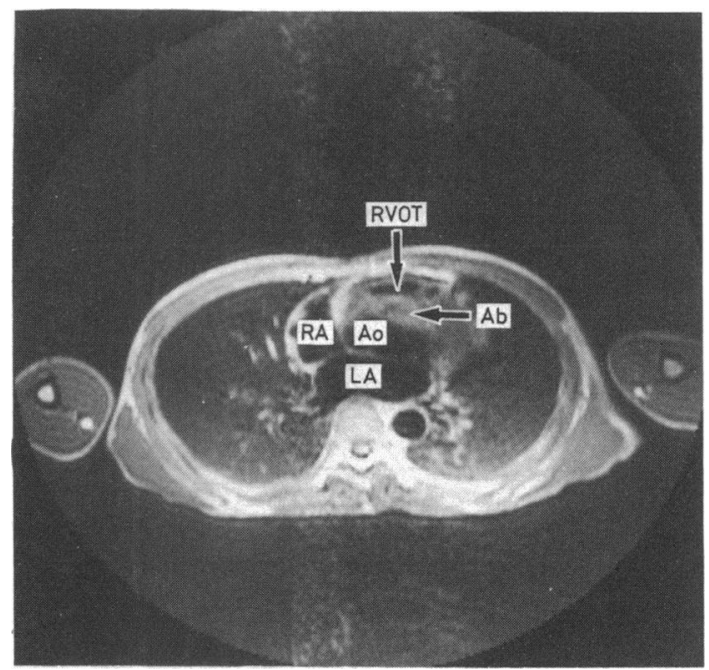

Fig 3 Magnetic resonance image of transverse section at the level of the aortic valve in a patient with infective endocarditis on a severely incompetent valve. There is an abscess mass $(A b)$ involving the anterior aortic wall and compressing the right ventricular outflow tract (RVOT). Ao, aortic root; $L A$, left atrium; $R A$, right atrium. 
significant $(\mathrm{p}<0.05)$, and the difference between the moderate and severe groups was highly significant $(p<0.001)$.

Two patients were restudied after valve operation. In one (preoperative image in fig 3 ) after homograft aortic root and valve replacement the stroke volume

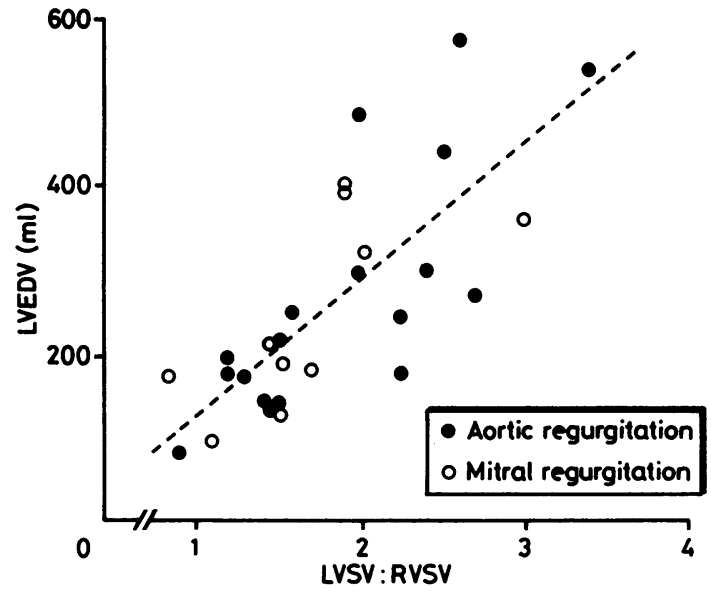

Fig 4 Correlation between the left ventricular end diastolic volume (LVEDV) and left to right ventricular stroke volume ratio ( $L V S V: R V S V$ ) measured by magnetic resonance. The dotted line shows the regression equation $(y=161 x-32, r=0.75$, standard error of the estimate $=88.8 \mathrm{ml}, p<0.001)$.

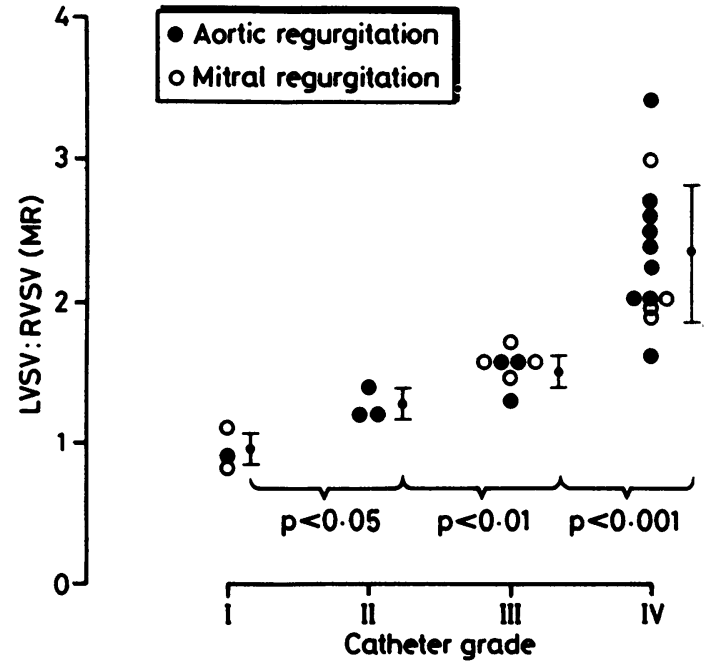

Fig 5 Magnetic resonance (MR) measurements of the ratio of left ventricular stroke volume to right ventricular stroke volume (LVSV:RVSV) compared with angiographic assessment of the severity of regurgitation. The bars represent mean and standard deviation in each group. ratio fell from 2.0 to 0.95 , and in the other after mitral valve repair it fell from 1.9 to 0.95 .

Figures 6 to 8 show the relation between magnetic resonance and radionuclide measurements of left ventricular ejection fraction, right ventricular ejection fraction, and stroke volume ratio. There was a

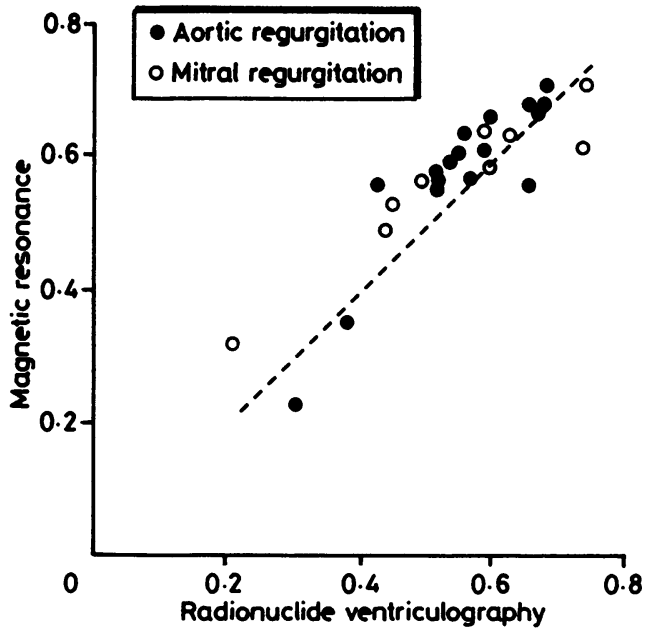

Fig 6 Correlation between magnetic resonance and radionuclide measurements of left ventricular ejection fraction $(y=0.73 x+0.16, r=0.85, p<0.001$, standard error of the estimate $=0.06)$. The dotted line is the line of identity.

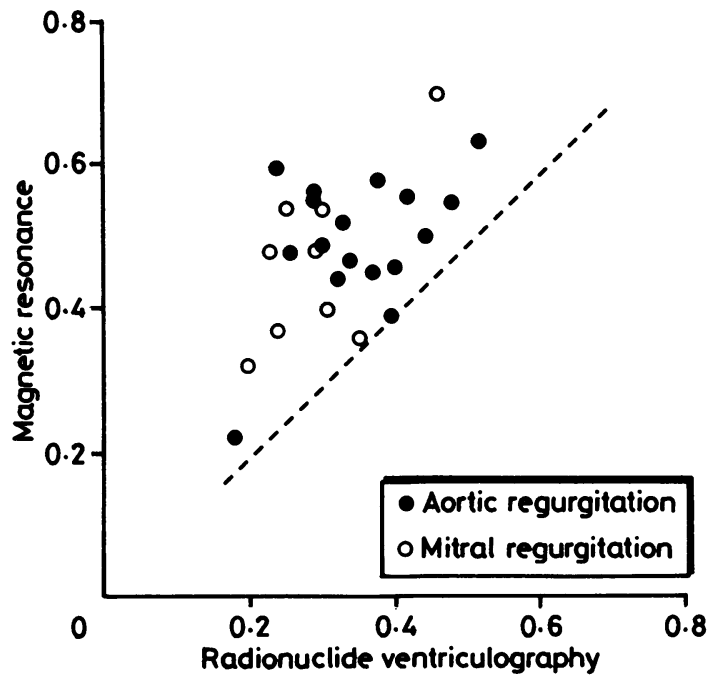

Fig 7 Correlation between magnetic resonance and radionuclide measurements of right ventricular ejection fraction $(y=0.69 x+0.25, r=0.58, p<0.01$, standard error of the estimate $=0.09)$. The dotted line is the line of identity. 


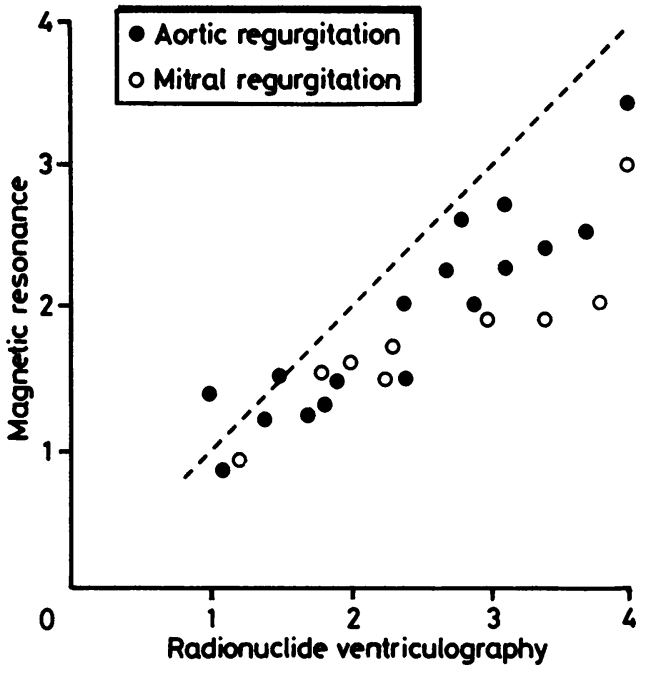

Fig 8 Correlation between magnetic resonance and radionuclide measurements of left to right ventricular stroke volume ratio $(y=0.61 x+0.36, r=0.88, p<0.001$, standard error of the estimate $=0.31$ ). The dotted line is the line of identity.

close relation for left ventricular ejection fraction (mean (SD) difference between measurements = $0.012(0.070)$ ), but for right ventricular ejection fraction it was much worse, with the radionuclide method consistently giving lower results (mean difference $=0.144(0.088))$. Agreement for the stroke volume ratio was good but the radionuclide method gave higher values than magnetic resonance $($ mean difference $=-0.620(0.464))$.

\section{Discussion}

Several methods are available for the assessment of valvar regurgitation, and although echocardiography is the most widely used imaging technique, without Doppler ultrasound quantification is difficult. We have chosen to compare magnetic resonance with angiocardiography and radionuclide ventriculography because they are most commonly used to assess the severity of regurgitation. There is in general good correlation, but the differences illustrate some of the problems of angiographic and radionuclide assessment.

\section{ANGIOCARDIOGRAPHY}

Angiocardiography is widely performed and although it is invasive its ability to measure pressure allows an assessment of stenosis as well as regurgitation. It is not suitable, however, for regular follow up, and it provides only a semiquantitative mea- surement which has eluded more accurate description. A more precise measurement can be obtained by comparing the forward stroke volume measured by thermodilution or indicator dilution with total stroke volume measured by left ventriculography but the results are not reproducible and their accuracy in routine use is poor. ${ }^{12}$

This study compares magnetic resonance measurements and the commonest angiographic method, with experienced observers each using the same criteria. The criteria essentially assess regurgitant volume, ${ }^{8-11}$ and although the volume of the chamber into which the contrast regurgitates may influence the findings the result will be closely related to regurgitant volume or fraction (regurgitant fraction = regurgitant volume $\div$ stroke volume). Since the left to right ventricular stroke volume ratio is related reciprocally to regurgitant fraction (regurgitant fraction $=1-1 \div$ stroke volume ratio), the shape of the relation in fig 5 can be predicted. It is expected that there will be a wide range of stroke volume ratios within the group with high regurgitant fractions (severe regurgitation) but narrower ranges in the less severe groups.

\section{RADIONUCLIDE VENTRICULOGRAPHY}

Radionuclide methods for the assessment of valvar regurgitation are non-invasive and they are suitable for regular follow up of adults ${ }^{1314}$ and children. ${ }^{1516}$ In addition, studies can be performed during dynamic exercise to provide information on the effects of regurgitation upon ventricular function. Both first pass ${ }^{1718}$ and equilibrium techniques have been used, ${ }^{1319}$ the latter more widely. Values obtained by equilibrium radionuclide ventriculography depend upon the methodology, and although the method used in this study gives reproducible results ${ }^{13}$ the stroke volume ratio is greater than 1 in normal subjects with a range of 1.0 to 1.7 (S R Underwood, S Walton, unpublished observations). This is because in the left anterior oblique projection (even with caudal tilt) the right ventricle and atrium overlap and right ventricular stroke counts are underestimated. ${ }^{20}$ Figure 8 shows this effect. The radionuclide technique consistently overestimates the stroke volume ratio, but correlation with magnetic resonance is good and the radionuclide value alone could be interpreted by reference to the regression equation or to a normal range.

Overlap between right atrium and ventricle also leads to an underestimation of right ventricular background counts and right ventricular ejection fraction. Figure 7 shows that the radionuclide measurement of right ventricular ejection fraction is consistently lower than the magnetic resonance 
measurement but, more importantly, correlation between the two is not as good as for the left ventricle. This implies that the contribution of the right atrium to background is very variable, and right ventricular ejection fraction measured by this technique should be interpreted with caution.

\section{MAGNETIC RESONANCE}

Volume measurements by magnetic resonance are very simple but there are several points to consider in achieving accurate results. Most important is image quality and patient cooperation is essential for this. ${ }^{21}$ In future imaging times will be reduced, simplifying the imaging of sick or uncooperative patients: real time imaging is already possible. ${ }^{22}$ Respiratory gating can be used to eliminate respiratory motion artefact, but imaging time is unacceptably prolonged and alternative techniques such as respiratory ordered phase encoding ${ }^{23}$ are more suitable.

Irregular rhythms also degrade image quality because the size and position of the heart may vary with each heart beat. Many patients with mitral valve disease are in atrial fibrillation, but satisfactory images are still obtained (fig 1). Because there is less variation in end systolic size of the ventricles the systolic images are better than diastolic. It is technically possible to reject data from beats with lengths outside a predetermined range if the aquisition time is extended. This is particularly helpful in patients with extrasystoles or profound sinus arrhythmia, but was not necessary in any of the patients in this study.

Accuracy in outlining the endocardium is also important and some experience is needed to achieve reproducible results. Visual methods are adequate for edge detection in these high resolution, high contrast images, but automatic edge detection would speed up the process. An automatic programme would need to be complex, since it can often be difficult to distinguish between signals from the myocardium and from static blood within the ventricle,${ }^{24}$ particularly under the anterior cusp of the tricuspid valve. In addition, criteria must be defined for the inclusion or exclusion of trabeculae and papillary muscles. We excluded papillary muscles but included small trabeculae in the cavity because the latter may not occupy the whole of a $10 \mathrm{~mm}$ slice, and we found that exclusion gave low values for right ventricular stroke volume. ${ }^{4}$ Thinner slices are more accurate but they increase the acquisition time or decrease the signal to noise ratio.

An important limitation of the use of the stroke volume ratio for the measurement of regurgitation is that in the presence of multiple valve disease or shunting the ratio reflects the sum of all effects. With equally severe mitral and tricuspid regurgitation for instance the ratio will be 1 . More accurate assessment of multiple lesions will be possible by magnetic resonance flow imaging. ${ }^{25} 26$ A colour coded cine display of blood velocity within the cardiac chambers and arteries may be superimposed upon the anatomical images, ${ }^{27}$ allowing an assessment of regurgitation in the same way as two dimensional Doppler flow imaging, ${ }^{28}$ although with greater versatility.

Some patients with valvar heart disease may have prosthetic valves but this is not a contraindication to magnetic resonance imaging. ${ }^{29}$ Virtually all modern valves are non-ferromagnetic and their workings are not affected by either static or changing magnetic fields. They may cause loss of magnetic resonance signal and hence a "black hole" in the image, but the area affected is small and does not prevent assessment of the rest of the heart.

\section{ECHOCARDIOGRAPHY}

Both $M$ mode and cross-sectional echocardiography are widely used in the assessment of patients with valvar heart disease. The presence and type of disease are seen and the severity of stenosis can be assessed. Valvar regurgitation is less easy to quantify, however, and reliance is usually placed on indirect observations such as relative chamber sizes and diastolic fluttering of the mitral valve. Continuous wave and pulsed Doppler echocardiography are sensitive and specific in the detection of valvar regurgitation, ${ }^{30}{ }^{31}$ and they allow an assessment of the severity of regurgitation in various ways. ${ }^{32-34}$ Two dimensional Doppler flow imaging is also of potential value, although it is not yet widely practised and its clinical role is still to be established. ${ }^{2835-38}$

Although cross-sectional echocardiography was performed on all the patients in this study, with Doppler velocity measurements in some, we did not compare the magnetic resonance results with echocardiography because reliable quantitative information was not always achieved.

\section{Conclusion}

Magnetic resonance imaging allows an assessment of cardiac anatomy and ventricular function in patients with isolated aortic and mitral regurgitation. It provides an accurate measure of ventricular volume, ejection fraction, and the left to right ventricular stroke volume ratio, which can be used to quantify the severity of regurgitation. Associated anatomical features may be seen, but details of valve disease are not well shown. Although it is unlikely to displace conventional methods of assessment in the short term, magnetic resonance imaging is a valuable addition and serves as a suitable standard by which to 
judge their accuracy. Improved resolution, rapid imaging techniques, and flow imaging promise an important role for magnetic resonance in the future assessment of valvar heart disease.

We thank the following for financial support: The Board of Governors of the National Heart and Chest Hospitals, the Coronary Artery Disease Research Association (CORDA), the League of Friends of The Brompton Hospital, the Viscount Royston Trust, the Halley Stewart Trust, the Charles Wolfson Charitable Foundation, and Picker International.

\section{References}

1 Steiner RE, Bydder GM, Selwyn A, et al. Nuclear magnetic resonance imaging of the heart: current status and future prospects. $\mathrm{Br}$ Heart $\mathcal{F} 1983 ; 50: 202-8$.

2 Higgins CB, Byrd BF, McNamara MT, et al. Magnetic resonance imaging of the heart: a review of the experience in 172 subjects. Radiology 1985;155:671-9.

3 Higgins CB, Kaufman L, Crooks LE. Magnetic resonance of the cardiovascular system. Am Heart $\mathcal{f}$ 1985;109:136-52.

4 Longmore DB, Klipstein RH, Underwood SR, et al. Dimensional accuracy of magnetic resonance in studies of the heart. Lancet 1985;i:1360-2.

5 Underwood SR, Klipstein RH, Firmin DN, et al. Magnetic resonance quantification of atrial shunting and valvar regurgitation [Abstract]. $\mathrm{Br}$ Heart $\mathcal{f}$ 1985; 54:616.

6 Pavel DG, Zimmer AM, Patterson VN. In vivo labelling of red blood cells with $99 \mathrm{~m}-\mathrm{Tc}$ : a new approach to blood pool visualization. $f \mathrm{Nucl}$ Med 1977; 18:305-8.

7 Geffers H, Adam WE, Bitter F, Siegel H, Kampmann $H$. Data processing and functional imaging in radionuclide ventriculography. In: Brill AB, Pryce RR, eds. Information processing in medical imaging. Proceedings of the 5th international conference. Nashville: Biomedical Computing Technology Information Center, 1977:322-32.

8 Mennel RG, Joyner CR Jr, Thompson PD, Pyle RR, MacVaugh $H$ III. The preoperative and operative assessment of aortic regurgitation. $\mathrm{Am} \mathcal{F}$ Cardiol 1972;29:360-6.

9 Hunt D, Baxley WA, Kennedy JW, Judge TP, Williams JE, Dodge HT. Quantitative evaluation of cine aortography in the assessment of aortic regurgitation. Am $\mathcal{F}$ Cardiol 1973;31:696-700.

10 Steiner RE, Jacobson G, Dinsmore R, Parizel G. Mitral regurgitation. Clin Radiol 1963;14:113-25.

11 Rees RSO, Jefferson KE, Harris AM. Cineangiocardiography of the mitral valve. $\mathrm{Br}$ Heart $\mathcal{f}$ 1965;27:498-504.

12 Mackenzie JD, Haites NE, Rawles JM. Method of assessing the reproducibility of blood flow measurement: factors influencing the performance of thermodilution cardiac output computers. Br Heart $\mathcal{f}$
1986;55:14-24.

13 Rigo P, Alderson PO, Robertson RM, Becker LC, Wagner HN Jr. Measurement of aortic and mitral regurgitation by gated cardiac blood pool scans. Circulation 1979;60:306-12.

14 Alderson PO. Radionuclide quantification of valvular regurgitation. $\mathcal{F}$ Nucl $\mathrm{Med}$ 1982;23:851-5.

15 Hurwitz RA, Treves S, Freed M, Girod DA, Caldwell RL. Quantitation of aortic and mitral regurgitation in the pediatric population: evaluation by radionuclide angiocardiography. Am $\mathcal{F}$ Cardiol 1983;51:252-5.

16 Parrish MD, Graham TP Jr, Born ML, et al. Radionuclide stroke count ratios for assessment of right and left ventricular volume overload in children. $A m \mathcal{F}$ Cardiol 1983;51:261-4.

17 Kirch DL, Metz CE, Steele PP. Quantitation of valvular insufficiency by computerised radionuclide angiocardiography. Am $\mathcal{F}$ Cardiol 1974;34:711-21.

18 Walton S, Underwood SR, Ell PJ, et al. Measurement of valvular regurgitation by first pass radionuclide ventriculography [Abstract]. Br Heart $\mathcal{F}$ 1984;52:91.

19 Nicod P, Corbett JR, Firth BG, et al. Radionuclide techniques for valvular regurgitation index: comparison in patients with normal and depressed ventricular function. $\mathcal{F} \mathrm{Nucl} \mathrm{Med} \mathrm{1982;23:763-9.}$

20 Berthout P, Cardot JC, Baud M, et al. Factors influencing the quantification of valvular regurgitation by gated equilibrium radionuclide angiography. Eur $\mathcal{f}$ Nucl Med 1984;9:112-4.

21 Schultz CL, Alfidi RJ, Nelson AD, Kopiwoda SY, Clampitt ME. The effect of motion on twodimensional Fourier transformation on magnetic resonance images. Radiology 1984;152:117-21.

22 Rzedzian R, Mansfield P, Doyle M, et al. Real-time nuclear magnetic resonance clinical imaging in paediatrics. Lancet 1983;ii:1281-2.

23 Bailes DR, Gilderdale DJ, Bydder GM, Collins AG, Firmin DN. Respiratory ordered phase encoding (ROPE): a method for reducing respiratory motion artefacts in MR imaging. $\mathcal{F}$ Comput Assist Tomogr 1985;9:835-8.

24 Von Schulthess GK, Fisher M, Crooks LE, Higgins CB. Gated MR imaging of the heart: intracardiac signals in patients and healthy subjects. Radiology 1985;156:125-32.

25 Nayler GL, Firmin DN, Longmore DB. Cine MR blood flow imaging. $\mathcal{f}$ Comput Assist Tomogr 1986;10:715-22.

26 Underwood SR, Firmin DN, Klipstein RH, Rees RSO, Longmore DB. Magnetic resonance velocity mapping: clinical application of a new technique [Abstract]. Proceedings of the Fifth Annual Meeting. Berkeley: Society of Magnetic Resonance in Medicine 1986;5:49-50.

27 Klipstein RH, Firmin DN, Underwood SR, et al. Magnetic resonance anatomical and blood flow information displayed in a single colour coded cine image. $\mathrm{Br}$ f Radiol 1986;59 (in press).

28 Omoto R, Yokote Y, Takamoto S, et al. The development of real-time two-dimensional Doppler echocardiography and its clinical significance in acquired valvular diseases: with special reference to the evalu- 
ation of valvular regurgitation. fpn Heart $f$ 1984;25:325-40.

29 Soulen R, Higgins CB, Budinger TF. Magnetic resonance imaging of prosthetic heart valves. Radiology 1985;154:705-7.

30 Come PC. The optimal Doppler examination: pulsed, continuous wave or both? $\mathcal{f} \mathrm{Am}$ Coll Cardiol 1986;7:886-8.

31 Wilde P, Pitcher D. Pulsed doppler echocardiography in cardiac diagnosis. $\mathcal{F} R$ Coll Physicians Lond 1986;20:25-31.

32 Pons-Llado G, Carreras-Costa F, Ballester-Rodes M, Auge-Sampera JM, Crexells-Figueras C, OriolPalou A. Pulsed Doppler patterns of left atrial flow in mitral regurgitation. Am $\mathcal{F}$ Cardiol 1986;57:806-10.

33 Masuyama T, Kodama K, Kitabatake A, et al. Noninvasive evaluation of aortic regurgitation by continuous-wave Doppler echocardiography. Circulation 1986;73:460-6.

34 Zhang Y, Nitter-Hauge S, Ihlen H, Rootwelt K, Myhre
E. Measurement of aortic regurgitation by Doppler echocardiography. Br Heart $\mathcal{F}$ 1986;55:32-8.

35 Miyatake K, Izumi S, Okamoto $M$, et al. Semiquantitative grading of severity of mitral regurgitation by real-time two-dimensional doppler flow imaging technique. $\mathcal{F} \mathrm{Am}$ Coll Cardiol 1986;7:82-8.

36 Suzuki Y, Kambara H, Kadota K, et al. Detection and evaluation of tricuspid regurgitation using a realtime, two-dimensional, color-coded, Doppler flow imaging system: comparison with contrast twodimensional echocardiography and right ventriculography. Am f Cardiol 1986;57:811-5.

37 Nakagawa H, Yamamoto K, Takamiya M, Sakakibara $\mathrm{H}$, Nimura Y. Semiquantitative grading of severity of mitral regurgitation by real-time two-dimensional doppler flow imaging technique. $\mathcal{F} \mathrm{Am}$ Coll Cardiol 1986;7:82-8.

38 Goldman ME. Real-time two-dimensional doppler flow imaging: a word of caution. $\mathcal{F} \mathrm{Am}$ Coll Cardiol 1986;7:89-90. 